\title{
Reduced relative dose intensity of primary chemotherapy does not influence prognosis of patients with Hodgkin lymphoma
}

\author{
Ludek Raidaa , Tomas Papajika, Zuzana Rusinakovaa, Vit Prochazkaa ${ }^{a}$ Edgar Faber ${ }^{\mathrm{a}}$, Dana Cahovab ${ }^{\mathrm{b}}$, Pavel Tucek ${ }^{\mathrm{b}}$, Karel Indrak ${ }^{\mathrm{a}}$
}

Aims. A retrospective analysis of patients with Hodgkin lymphoma $(\mathrm{HL})$ was performed to assess their outcome regarding relative dose intensity (RDI) of chemotherapy administered in primary treatment.

Methods. A total of 194 patients were divided into three groups with different RDI of primary chemotherapy (100\%, $90-$ $99 \%$ and < $90 \%)$. Reduced RDI in two groups (90-99\% and < $90 \%)$ was caused by the delay of the interval between the administration of some chemotherapeutic courses. The probability of complete remission (CR), disease relapse, eventfree survival (EFS) and overall survival (OS) as the basic parameters of patient outcome were statistically compared.

Results. Multivariate analysis showed here were no significant differences in probability of $\mathrm{CR}(\mathrm{HR} 0.9,95 \% \mathrm{Cl}[0.75-$ 1.08], $P=0.5)$, risk of relapse (HR 1.34, 95\% Cl [0.92-1.94], $P=0.11)$ or death (HR 1.52, 95\% Cl $[0.94-2.5], P=0.13)$. There were also no significant differences in probability of EFS (mean 13 vs. 10 vs. 12 years, $P=0.17 ; \mathrm{HR} 1.54,95 \% \mathrm{Cl}[0.91-2.6]$, $P=0.22$ ) or OS (mean 15 vs. 13 vs. 14 years, $P=0.13 ; \mathrm{HR} 1.52,95 \% \mathrm{Cl}[0.93-2.5], P=0.13$ ).

Conclusion. We found no significant impact of primary chemotherapy delay resulting in reduced RDI on outcome in HL patients.

Keywords: Hodgkin lymphoma, primary treatment, chemotherapy, relative dose intensity, therapeutic outcome

Received: April 24, 2012; Accepted with revision: March 20, 2013; Available online: April 10, 2013

http://dx.doi.org/10.5507/bp.2013.022

${ }^{a}$ Department of Hemato-Oncology, Faculty of Medicine and Dentistry, Palacky University Olomouc, Czech Republic

${ }^{b}$ Department of Geoinformatics, Faculty of Science, Palacky University Olomouc

Corresponding author: Ludek Raida, e-mail: raida@fnol.cz

\section{INTRODUCTION}

Over the last decades, the therapeutic outcome of Hodgkin lymphoma (HL) patients has dramatically improved. Combined chemotherapy has allowed the treatment of a significant number of patients with advanced disease. Nevertheless, about a half of those patients either fail to achieve complete remission (CR) with primary treatment or relapse. Intensified first-line chemotherapeutic regimens have been designed and used to overcome the risk of early malignant clone chemoresistance development. Rapid tumor burden reduction along with minimizing its regrowth during treatment might decrease the probability of malignant cell mutations leading to chemoresistance. The escalation of cytostatic doses and the shortening of time interval between their administration represent approaches to intensification based on mathematical modelling ${ }^{1-3}$. Examples of such intensified chemotherapeutic regimens in the primary treatment of HL are Stanford V and BEACOPP, particularly its escalated variant ${ }^{4-5}$. Unfortunately, the toxicity of intensive approaches is relatively high and the associated complications can delay the administration of other chemotherapeutic courses or lead to the reduction of cytostatic drug dose. Nevertheless, delay in chemotherapy without reducing scheduled doses is not unusual in patients treated with standard protocols (e.g. ABVD, various hybrid or alternative regimens) as well. The major reasons are infec- tions resulting not only from neutropenia that is usually less pronounced during standard therapy, but also from the immunodeficiency of HL patients ${ }^{6}$. Thus, the relative dose intensity (RDI) of primary chemotherapy can be reduced in a significant proportion of patients, even those in the early stage of disease and treated with less intensive protocols.

According to our knowledge there is no published study evaluating the delay of primary chemotherapy administration leading to the reduction of its RDI as a significant violation of treatment protocol with potential prognostic impact on patients suffering from HL.

\section{PATIENTS AND METHODS}

The main objective of the study was to compare the outcome of HL patients with respect to the RDI of chemotherapy administered in primary treatment. The achievement of CR, the risk of disease relapse and the death from any cause were assessed as the major endpoints of outcome. The data of 194 patients diagnosed with HL and treated in one centre between the years 1992 and 2005 were analysed; the median follow-up from diagnosis was 8 (range 1-17) years. All patients underwent chemotherapy in primary treatment and the differences between them including the use of various first-line chemotherapeutic protocols were evident. 


\section{Primary treatment}

The primary chemotherapeutic regimens were chosen with respect to the stage of disease. The patients diagnosed in early stages of disease (Table 1) were treated with two cycles of ABVD (Table 2). The majority of intermediate stages (Table 1) underwent treatment according to the Stanford V protocol ${ }^{4}$. Two cycles of COPP/ABVD were administered in six patients treated in the early 1990s. The treatment with COPP was used in two elderly patients suffering from the intermediate stage of HL (Table 2). BEACOPP regimens were used in 83 patients (Table 2) with advanced disease (Table 1). Less intensive hybrid chemotherapy $\mathrm{C}(\mathrm{M}) \mathrm{OPP} / \mathrm{ABV}$ and the time intensified protocol Stanford $\mathrm{V}$ were previously administered in 26 patients (Table 2). An involved-field radiotherapy (RT) of 30 Gy was applied either as the standard part of combined modality therapeutic protocols (all early stages, the intermediate ones treated with two cycles of COPP/ABVD, all intermediate and advanced stages with nodal bulk treated with Stanford V) or as adjuvant treatment targeted to the residual disease after first-line chemotherapy (intermediate stages without nodal bulk treated with Stanford V and patients with advanced disease and suspected of residual active HL) (Table 2). 4-6 weeks after the completion of

Table 1. The basic characteristics of HL patients by RDI.

\begin{tabular}{|c|c|c|c|c|}
\hline & RDI $100 \%(n=95)$ & RDI 90-99\% (n=53) & RDI < $90 \%(n=46)$ & $P$ \\
\hline female / male & $49(52 \%) / 46(48 \%)$ & $28(53 \%) / 25(47 \%)$ & $24(52 \%) / 22(48 \%)$ & 0.98 \\
\hline age (median) & $28(18-72)$ years & $28(18-74)$ years & $29(19-71)$ years & 0.92 \\
\hline \multicolumn{5}{|l|}{ histological subtype: } \\
\hline nodular sclerosis & $62(65 \%)$ & $32(60 \%)$ & $26(57 \%)$ & \multirow{3}{*}{0.012} \\
\hline mixed cellularity & $30(32 \%)$ & $21(40 \%)$ & $13(28 \%)$ & \\
\hline other subtypes & $3(3 \%)$ & 0 & $7(15 \%)$ & \\
\hline \multicolumn{5}{|l|}{ clinical stage: } \\
\hline I & $5(5 \%)$ & $4(8 \%)$ & $3(7 \%)$ & \multirow{5}{*}{0.033} \\
\hline II & $57(60 \%)$ & $32(60 \%)$ & $22(48 \%)$ & \\
\hline III & $26(27 \%)$ & $12(23 \%)$ & $8(17 \%)$ & \\
\hline IV & $7(8 \%)$ & $5(9 \%)$ & $13(28 \%)$ & \\
\hline early stages & $5(5 \%)$ & $1(2 \%)$ & $6(13 \%)$ & \\
\hline intermediate stages & $37(39 \%)$ & $21(39 \%)$ & $13(28 \%)$ & \multirow[t]{2}{*}{0.19} \\
\hline advanced stages & $53(56 \%)$ & $31(59 \%)$ & $27(59 \%)$ & \\
\hline B symptoms & $41(43 \%)$ & $26(57 \%)$ & $25(47 \%)$ & 0.33 \\
\hline nodal bulk $(\geq 5 \mathrm{~cm})$ & $60(63 \%)$ & $21(46 \%)$ & $32(60 \%)$ & 0.13 \\
\hline ESR (median) & $44(2-130) \mathrm{mm} / \mathrm{h}$ & $49(2-135) \mathrm{mm} / \mathrm{h}$ & $53(4-132) \mathrm{mm} / \mathrm{h}$ & 0.37 \\
\hline hemoglobin (median) & $132(93-171) \mathrm{g} / \mathrm{L}$ & $124(77-160) \mathrm{g} / \mathrm{L}$ & $122(88-165) \mathrm{g} / \mathrm{L}$ & 0.016 \\
\hline albumin (median) & $38.8(26-50.8) \mathrm{g} / \mathrm{L}$ & $39.8(17.9-49.8) \mathrm{g} / \mathrm{L}$ & $37.4(21.7-46.3) \mathrm{g} / \mathrm{L}$ & 0.045 \\
\hline WBC (median) & $10.0(4.3-23.1) \times 10^{9} / \mathrm{L}$ & $9.1(3.8-21.8) \times 10^{9} / \mathrm{L}$ & $8.35(3.3-20.0) \times 10^{9} / \mathrm{L}$ & 0.14 \\
\hline ALC (median) & $1.55(0.1-4.1) \times 10^{9} / \mathrm{L}$ & $1.45(0-4.0) \times 10^{9} / \mathrm{L}$ & $1.21(0.2-3.9) \times 10^{9} / \mathrm{L}$ & 0.088 \\
\hline
\end{tabular}

Table 2. The primary treatment of HL patients by RDI.

\begin{tabular}{|c|c|c|c|c|}
\hline & RDI $100 \%(n=95)$ & RDI $90-99 \%(n=53)$ & $\mathrm{RDI}<90 \%(\mathrm{n}=46)$ & $P$ \\
\hline \multicolumn{5}{|l|}{ chemotherapeutic regimen: } \\
\hline ABVD $2 x$ & $5(5 \%)$ & $1(2 \%)$ & $6(11 \%)$ & \\
\hline COPP 8x & $2(2 \%)$ & 0 & 0 & \\
\hline $\mathrm{C}(\mathrm{M}) \mathrm{OPP} / \mathrm{ABV} 6 \mathrm{x}$ & $7(7 \%)$ & $3(6 \%)$ & $3(6 \%)$ & \\
\hline COPP/ABVD 2x & $2(2 \%)$ & $3(6 \%)$ & $1(2 \%)$ & 0.59 \\
\hline Stanford V & $41(43 \%)$ & $22(42 \%)$ & $15(28 \%)$ & \\
\hline BEACOPP (B) 8x & $18(19 \%)$ & $13(25 \%)$ & $8(15 \%)$ & \\
\hline BEACOPP (E) 8x & $16(17 \%)$ & $9(17 \%)$ & $9(17 \%)$ & \\
\hline $\operatorname{BEACOPP}(\mathrm{E}+\mathrm{B}) 4 \mathrm{x}+4 \mathrm{x}$ & $4(4 \%)$ & $2(4 \%)$ & $4(8 \%)$ & \\
\hline $\mathrm{RT}$ in primary treatment & $37(39 \%)$ & $15(28 \%)$ & $23(50 \%)$ & 0.54 \\
\hline
\end{tabular}

ABVD - 28-day regimen with adriamycin, bleomycin, vinblastine, dacarbazine, COPP - 21-day regimen with cyclophosphamide, vincristine, procarbazine, prednisone, C(M)OPP/ABV - 28-day hybrid regimens with cyclophosphamide (or mustargen), vincristine, procarbazine, prednisone, adriamycin, bleomycin, vinblastine, COPP/ABVD - 56-day alternative regimen with cyclophosphamide, vincristine, procarbazine, prednisone, adriamycin, bleomycin, vinblastine, dacarbazine, Stanford V - 12-week regimen with mustargen, adriamycin, etoposide, bleomycin, vincristine, vinblastine, prednisone, BEACOPP (B) - 21-day baseline regimen with bleomycin, etoposide, adriamycin, cyclophosphamide, vincristine, procarbazine, prednisone, BEACOPP (E) - 21-day regimen with bleomycin, vincristine, procarbazine, prednisone and the escalated doses of cyclophosphamide, adriamycin and etoposide 
chemotherapeutic protocol a therapeutic response in those patients considered for adjuvant RT was assessed either by computed tomography (CT) combined with gallium scintigraphy, magnetic resonance imaging (MRI) (ref. ${ }^{7}$ ) or more recently by $18 \mathrm{~F}$-flourodeoxyglucose positron emission tomography-computed tomography (FDGPET-CT). The patients without any evidence of active tumour were considered to have achieved complete remission (CR) of disease and they were further observed without adjuvant RT. Involved field RT was administered if there had been any suspicion of residual active disease. A definitive response to the first-line treatment was evaluated at least 8 weeks after the completion of RT regardless if primarily planned or adjuvant.

\section{Relative dose intensity of chemotherapy}

All patients received the total doses of cytostatics strictly according to the scheduled protocol but interruptions and delays in chemotherapy treatment were found in 99 ( $51 \%$ of them). Despite the appropriate dose density (DD; defined as the drug dose per square meter) of chemotherapy according to the protocol, the delay of treatment led to the reduction in dose intensity (DI; defined as the drug dose per square meter per day). The RDI was calculated as the quota of real DI administered to the patient in primary treatment and scheduled DI according to the chemotherapeutic protocol. The median of reduced RDI in those 99 patients was 89\% (range 43-99\%) and became the cut-off level for dividing them into two groups. Thus, all 194 patients were divided into three cohorts: 95 (49\%) with an RDI of $100 \%, 53(27 \%)$ with an RDI of 90-99\% and 46 (24\%) with an RDI < 90\%.

\section{Statistical analysis}

Those three cohorts were statistically compared. The differences in non-metric parameters (gender, histological subtypes, disease clinical stage, the manifestation of B symptoms, lymph node bulk, the use of various chemotherapeutic protocols and RT in primary treatment) were analyzed by the chi-square test. Age, initial erythrocyte sedimentation rate (ESR), albumin and hemoglobin levels, white blood cells (WBC) and absolute lymphocyte count (ALC) as the metric parameters were compared using the Kruskal-Wallis test. The therapeutic response was compared between the three RDI groups using the evaluation of CR numbers, the incidence of relapses and deaths using univariate analysis (chi-square test). The probability of CR achievement, the risk of disease relapse and death were analyzed by the Cox proportional hazards $\operatorname{model}^{8}$ as the method of multivariate analysis. The basic characteristics of disease (Table 1) and primary treatment (Table 2) found to be significantly different in the three compared RDI groups were included besides RDI as the variables into that analysis. The Kaplan-Meier curves ${ }^{9}$ of event-free survival (EFS; assessed in patients who achieved CR with primary treatment and defined as the period between the confirmation of remission and disease relapse or death from any cause) and overall survival (OS; defined as the period between the time of HL diagnosis and death from any cause) were calculated and constructed. The differences between the three variables were compared using the log-rank test in univariate analysis and Cox proportional hazards model in multivariate one.

\section{RESULTS}

There were statistically significant differences between the three RDI groups in histological subtypes of $\mathrm{HL}$ $(P=0.012)$, clinical stage of disease $(P=0.033)$, hemoglobin $(P=0.016)$ and albumin $(P=0.045)$ levels (Table 1$)$. Other histological subtypes than nodular sclerosis and mixed cellularity classical HL as well as the advanced clinical stages III and IV were more frequently observed among the patients with RDI < 90\% (Table 1). Nevertheless, no differences between the groups were found in numbers of patients with early, intermediate and advanced disease (Table 1). Tendency to higher initial hemoglobin levels was found in the group with RDI 100\% compared to patients treated with RDI 90-99\% and < 90\% (Table 1). Significantly lower albumin levels were measured in patients who underwent primary chemotherapy with RDI < $90 \%$ (Table 1). On the other hand, no differences between the RDI groups were found for patient gender and age, manifestation of B symptoms, nodal bulk, ESR, WBC, ALC (Table 1), chemotherapeutic protocols and RT used in the primary treatment of HL (Table 2). Histological subtypes, clinical stage, hemoglobin and albumin levels as significantly different parameters in the three RDI groups became the other variables included in the multivariate analysis.

Table 3. The therapeutic outcome of HL patients by RDI.

\begin{tabular}{|c|c|c|c|c|c|c|c|}
\hline & RDI $100 \%(n=95)$ & $\begin{array}{l}\text { RDI } 90-99 \% \\
\quad(n=53)\end{array}$ & $\begin{array}{c}\text { RDI }<90 \% \\
\quad(n=46)\end{array}$ & $P_{\mathrm{UVA}}$ & HR & $95 \% \mathrm{CI}$ & $P_{\mathrm{MVA}}$ \\
\hline $\mathrm{CR}$ & $87(92 \%)$ & $49(93 \%)$ & $40(87 \%)$ & 0.62 & 0.9 & $0.75-1.08$ & 0.5 \\
\hline relapses & $15(17 \%)$ & $15(31 \%)$ & $10(25 \%)$ & 0.18 & 1.34 & $0.92-1.94$ & 0.11 \\
\hline deaths & $8(8 \%)$ & $5(9 \%)$ & $10(22 \%)$ & 0.075 & 1.52 & $0.93-2.5$ & 0.13 \\
\hline $\begin{array}{l}\text { EFS } \\
\text { (mean) }\end{array}$ & $\begin{array}{c}13 \text { years * } \\
95 \% \text { CI }[12-14]\end{array}$ & $\begin{array}{c}10 \text { years * } \\
95 \% \text { CI [8-12] }\end{array}$ & $\begin{array}{c}12 \text { years * } \\
95 \% \text { CI }[10-14]\end{array}$ & 0.17 & 1.54 & $0.91-2.6$ & 0.22 \\
\hline $\begin{array}{l}\text { OS } \\
\text { (mean) }\end{array}$ & $\begin{array}{c}15 \text { years * } \\
95 \% \text { CI [14-16] }\end{array}$ & $\begin{array}{c}13 \text { years * } \\
95 \% \text { CI [12-14] }\end{array}$ & $\begin{array}{c}14 \text { years * } \\
95 \% \text { CI [12-15] }\end{array}$ & 0.13 & 1.52 & $0.93-2.5$ & 0.13 \\
\hline
\end{tabular}

\footnotetext{
* median was not achieved, UVA - univariate analysis, MVA - multivariate analysis
} 


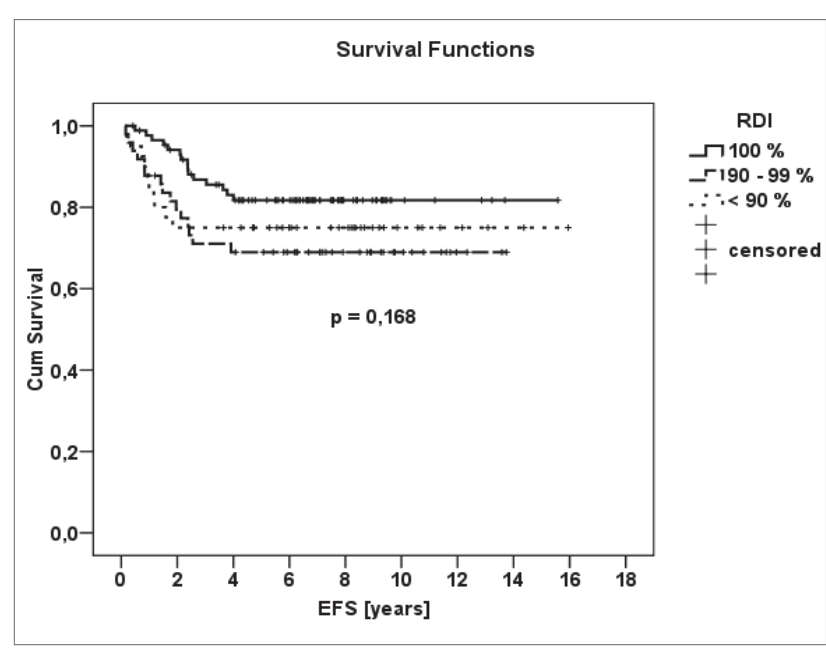

Fig. 1. The Kaplan-Meier curves of event-free survival by RDI.

A total of 176 of 194 patients (91\%) achieved the CR of $\mathrm{HL}$ with the first-line therapy. Of those, forty $(23 \%)$ relapsed and 23 patients (12\%) died during the follow-up period. There were no significant differences between the RDI groups in rates and probability of CR, relapses and deaths including EFS and OS according to both univariate and multivariate analyses (Table 3, Fig. 1, 2).

\section{DISCUSSION AND CONCLUSION}

The dose intensity of primary chemotherapy is assumed to play an important role in tumor control and elimination ${ }^{10}$. Nevertheless, despite the use of growth factors to shorten the neutropenic period and severity of anemia, primary chemotherapy has to be delayed or reduced in a significant number of patients. In particular active infections can contribute to the violation of primary chemotherapeutic protocol administration. Several studies evaluating the RDI of chemotherapy used in the primary treatment of HL have already been published. Swedish authors analyzed the impact of primary chemotherapy RDI on the OS and case-specific survival (CSS) of 88 elderly (> 60 years old) patients. The cut-off of RDI was $65 \%$. The patients with RDI $>65 \%$ had significantly better OS and CSS than those with RDI $\leq 65 \%$ (ref. ${ }^{11}$ ). However, the $\mathrm{RDI} \leq 65 \%$ may be considered as significant violation of primary chemotherapy that is considered to decrease its therapeutic efficacy. Undoubtedly, those results are valuable for seeking an optimal therapeutic approach to the limited group of elderly HL patients. Nevertheless, the reduction of primary chemotherapy RDI $\geq 35 \%$ is unusual in the majority of patients diagnosed with HL. In their study, Owadally et al. analyzed 380 patients suffering from advanced $\mathrm{HL}$ and treated with ABVD. They were divided into three primary chemotherapy RDI groups (> $97 \%, 86-97 \%$ and < 86\%). Multivariate analysis showed no significant influence of RDI on progression-free survival (PFS) or OS (ref. ${ }^{12}$ ). Lower RDIs in those two studies were not only the results of chemotherapy delay but

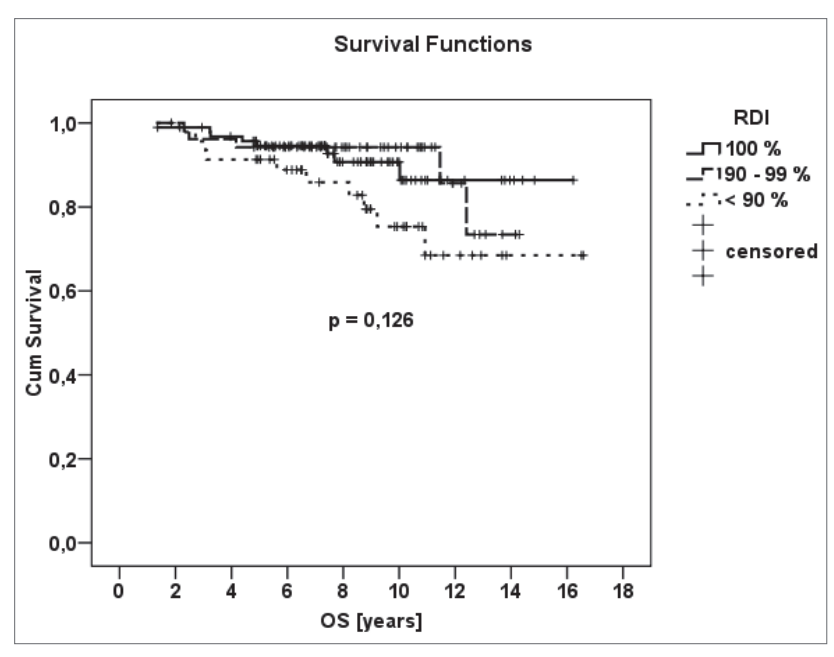

Fig. 2. The Kaplan-Meier curves of overall survival by RDI.

also, in contrast to our present study, the reduction of cytostatic doses. Chemotherapy intensification has usually been based on the Skipper model ${ }^{1-2}$ resulting in the design of protocols with cytostatic dose escalation and the shortening of interval between their administration. Nevertheless, according to mathematical modelling published by Hasenclever et al., dose escalation should be more important for HL patient outcome than the shortening of treatment duration ${ }^{3}$. Lagarde et al. supported that hypothesis in a relatively small study more than twenty years ago. He assessed the outcome of $95 \mathrm{HL}$ patients in clinical stages I-IIIA and treated with CVPP chemotherapy (cyclophosphamide, vinblastine, procarbazine and prednisone) followed by extended-field RT regarding to the DI and DD of the used cytostatics. Better diseasefree survival (DFS) correlated with higher DD but not DI $\left(\right.$ ref. $\left.{ }^{13}\right)$. Fourteen years later Diehl et al. also confirmed the validity of mathematical modellling in a randomized study comparing the primary treatment of advanced stage HL with COPP/ABVD, baseline and escalated BEACOPP regimen ${ }^{14}$.

The aim of the present study was to analyze the impact of primary chemotherapy delay resulting in reduced RDI on the outcome of various HL patients treated according to appropriate disease stage adjusted protocols. A half of the patients in the study underwent primary treatment with reduced cytostatics RDI $(<100 \%)$ because of the chemotherapy delay. The RDI median of $89 \%$ in that group became the cut-off for further analysis. Using the approach of dividing the patients with HL into three groups according to the RDI of primary chemotherapy (RDI $100 \%$, RDI $90-99 \%$ and RDI $<90 \%$ ), there was no confirmed influence of primary chemotherapy dose intensity on numbers of CR, relapses and deaths measured as probability, EFS and OS by either univariate or multivariate analysis. Despite the heterogenous cohort of patients, our results support some previously published observations concerning reduction in chemotherapy RDI in the primary treatment of HL. 


\section{ACKNOWLEDGEMENTS}

This work was supported by the grant MSM 6198959205 of the Ministry of Education, Youth and Sports of the Czech Republic, projects LF-2012-007 and PrF_2013_024 of Palacky University in Olomouc. The authors also acknowledge the support by the Operational Program Education for Competitiveness - European Social Fund (project CZ.1.07/2.3.00/20.0170 of the Ministry of Education, Youth and Sports of the Czech Republic).

Authorship contributions: LR, TP, VP: literature search; LR: study design; LR, TP, ZR, VP, EF: data collection; LR, DC, PT: data analysis; LR: data interpretation; DC, PT: statistical analysis, figures; LR: manuscript writing; TP, ZR, VP, EF, KI: final approval.

Conflict of interest statement: The authors stated that there are no conflicts of interest regarding the publication of this article.

\section{REFERENCES}

1. Skipper HE, Schabel FM, Wilcox WS. Experimental evaluation of potential anticancer agents. XII. On the criteria and kinetics associated with curability of experimental leukemia. Cancer Chemother Rep 1964;35:1-111.

2. Skipper HE, Schabel FM, Wilcox WS. Experimental evaluation of potential anticancer agents. XIV. Further studies on certain basic concepts underlying chemotherapy of leukemia. Cancer Chemother Rep 1965:45:5-28.

3. Hasenclever D, Brosteanu O, Gerike T, Loeffler M. Modelling of chemotherapy: The effective dose approach. Ann Hematol 2001;80:8994.

4. Horning SJ, Hoppe RT, Breslin S, Barlett NL, Brown BW, Rosenberg SA. Stanford V and radiotherapy for locally extensive and advanced
Hodgkin's disease: Mature results of a prospective clinical trial. J Clin Oncol 2002;20:630-7.

5. DiehI V, Franklin J, Hasenclever D, Tesch H, Pfreundschuh M, Lathan B, Paulus U, Sieber M, Rüffer JU, Sextro M, Engert A, Wolf J, Hermann R, Holmer L, Stappert-Jahn U, Winnerlein-Trump E, Wulf G, Krause $\mathrm{S}$, Glunz A, von Kalle K, Bischoff $\mathrm{H}$, Haedicke C, Dühmke E, Georgii A, Loeffler M. A new regimen for advanced Hodgkin's disease. Ann Oncol 1998:9:67-71.

6. Hillinger SM, Herzig GP. Impaired cell-mediated immunity in Hodgkin's disease mediated by suppressor lymphocytes and monocytes. J Clin Invest 1978;61:1620-7.

7. Heřman $M$, Pauček B, Raida L, Mysliveček $M$, Zapletalová J. Comparison of magnetic resonance imaging and (67)gallium scintigraphy in the evaluation of posttherapeutic residual mediastinal mass in the patients with Hodgkin's lymphoma. Eur J Radiol 2007;64:432-38.

8. Cox D. Regression models and life tables. J R Stat Soc (B) 1972;34:187220.

9. Kaplan E, Meier P. Nonparametric estimation from incomplete observations. J Am Stat Assoc 1957;53:457-81.

10. Goldie JH, Coldman AJ. A mathematic model for relating the drug sensitivity of tumors to their spontaneous mutation rate. Cancer Treat Rep 1979;63:1727-33.

11. Landgren O, Algernon C, Axdorph U, Nilsson B, Wedelin C, PorwitMacDonald A, Grimfors G, Björkholm M. Hodgkin's lymphoma in the elderly with special reference to type and intensity of chemotherapy in relation to prognosis. Haematologica 2003;88:438-44.

12. Owadally WS, Sydes MR, Radford JA, Hancock BW, Cullen MH, Stenning SP, Johnson PW. Initial dose intensity has limited impact on the outcome of ABVD chemotherapy for advanced Hodgkin lymphoma (HL): data from UKLG LY09 (ISRCTN97144519). Ann Oncol 2010;21:568-73.

13. Lagarde P, Bonichon F, Eghbali H, de Mascarel I, Chauvergne J, Hoerni $B$. Influence of dose intensity and density on therapeutic and toxic effects in Hodgkin's disease. Br J Cancer 1989;59:645-49.

14. DiehI V, Franklin J, Pfreundshuh M, Lathan B, Paulus U, Hasenclever D, Tesch H, Herrmann R, Dörken B, Müller-Hermelink HK, Dühmke E, Loeffler M. Standard and increased-dose BEACOPP chemotherapy compared with COPP-EBVD for advanced Hodgkin's disease. N Engl J Med 2003;348:2386-95. 